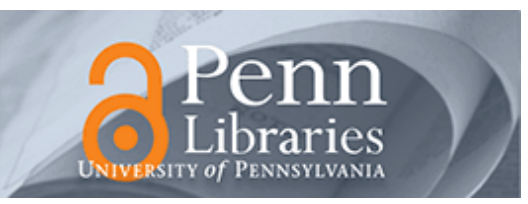

University of Pennsylvania

ScholarlyCommons

2-23-2010

\title{
Temperature Dependence of Thermal Conductivity Enhancement in Single-walled Carbon Nanotube/polystyrene Composites
}

\author{
Michael B. Jakubinek \\ Dalhousie University \\ Mary Anne White \\ Dalhousie University \\ Minfang Mu \\ University of Pennsylvania \\ Karen I. Winey \\ University of Pennsylvania, winey@lrsm.upenn.edu
}

Follow this and additional works at: https://repository.upenn.edu/mse_papers

Part of the Materials Science and Engineering Commons

\section{Recommended Citation}

Jakubinek, M. B., White, M., Mu, M., \& Winey, K. I. (2010). Temperature Dependence of Thermal Conductivity Enhancement in Single-walled Carbon Nanotube/polystyrene Composites. Retrieved from https://repository.upenn.edu/mse_papers/183

Suggested Citation:

Jakubinek, M.B., M. White, M. Mu and K.I. Winey. (2010). "Temperature dependence of thermal conductivity enhancement in single-walled carbon nanotube/polystyrene composites." Applied Physics Letters. Vol. 96, 083105

(C) 2010 American Institute of Physics. This article may be downloaded for personal use only. Any other use requires prior permission of the author and the American Institute of Physics. The following article appeared in Applied Physics Letters and may be found at http://dx.doi.org/10.1063/1.3323095

This paper is posted at ScholarlyCommons. https://repository.upenn.edu/mse_papers/183

For more information, please contact repository@pobox.upenn.edu. 


\title{
Temperature Dependence of Thermal Conductivity Enhancement in Single-walled Carbon Nanotube/polystyrene Composites
}

\author{
Abstract \\ The thermal conductivity of single-walled carbon nanotube (SWCNT)/polystyrene composites, prepared \\ by a method known to produce a uniform distribution of SWCNT bundles on the micrometer length scale, \\ was measured in the temperature range from approximately 140 to $360 \mathrm{~K}$. The thermal conductivity \\ enhancement ( $50 \%$ for 1 mass $\%$ at $300 \mathrm{~K}$ ) is reasonably constant above room temperature but is reduced \\ at the lower temperatures. This result is consistent with the expected, large contribution of interfacial \\ thermal resistance in SWCNT/polymer composites. Enhancements in electrical conductivity show that 1 \\ mass \% loading is in the region of the electrical percolation threshold.

\section{Disciplines} \\ Engineering | Materials Science and Engineering

\section{Comments} \\ Suggested Citation: \\ Jakubinek, M.B., M. White, M. Mu and K.I. Winey. (2010). "Temperature dependence of thermal \\ conductivity enhancement in single-walled carbon nanotube/polystyrene composites." Applied Physics \\ Letters. Vol. 96, 083105 \\ (C) 2010 American Institute of Physics. This article may be downloaded for personal use only. Any other \\ use requires prior permission of the author and the American Institute of Physics. The following article \\ appeared in Applied Physics Letters and may be found at http://dx.doi.org/10.1063/1.3323095
}




\title{
Temperature dependence of thermal conductivity enhancement in single-walled carbon nanotube/polystyrene composites
}

\author{
Michael B. Jakubinek, ${ }^{1}$ Mary Anne White, ${ }^{1, a)}$ Minfang $\mathrm{Mu}^{2}$ and Karen I. Winey ${ }^{2}$ \\ ${ }^{1}$ Departments of Physics and Chemistry, and Institute for Research in Materials, Dalhousie University, \\ Halifax, Nova Scotia B3H 4J3, Canada \\ ${ }^{2}$ Department of Materials Science and Engineering, University of Pennsylvania, Philadelphia, \\ Pennsylvania 19104-672, USA
}

(Received 22 September 2009; accepted 21 January 2010; published online 23 February 2010)

\begin{abstract}
The thermal conductivity of single-walled carbon nanotube (SWCNT)/polystyrene composites, prepared by a method known to produce a uniform distribution of SWCNT bundles on the micrometer length scale, was measured in the temperature range from approximately 140 to $360 \mathrm{~K}$. The thermal conductivity enhancement $(50 \%$ for 1 mass $\%$ at $300 \mathrm{~K}$ ) is reasonably constant above room temperature but is reduced at the lower temperatures. This result is consistent with the expected, large contribution of interfacial thermal resistance in SWCNT/polymer composites. Enhancements in electrical conductivity show that 1 mass \% loading is in the region of the electrical percolation threshold. (C) 2010 American Institute of Physics. [doi:10.1063/1.3323095]
\end{abstract}

Several current and proposed applications of carbon nanotubes (CNTs) involve their use as additives to improve the properties in polymer composites. ${ }^{1}$ The high electrical conductivity and thermal conductivity of individual CNTs as well as their excellent mechanical properties and high aspect ratios originally suggested that dramatic improvements in the properties of a polymer could be achieved by addition of relatively small amounts of CNTs. Indeed dramatic improvements (factors of $10^{10}$ to $10^{15}$ ) in electrical conductivity, $\sigma$, are routinely observed due to the formation of percolating networks of CNTs at very low loadings (often less than 0.1 mass \%). ${ }^{2,3}$ Large improvements in thermal conductivity, $\kappa$ (approximately 50-fold at 1 mass \% for randomly oriented CNTs), ${ }^{4}$ could be expected based on the dimensions, orientation, and intrinsic thermal conductivity of the CNTs. However, most experimental studies report enhancements of less than a factor of two for 1 mass \% CNTs and some report no enhancement or even a reduction in thermal conductivity relative to the polymer matrix. ${ }^{2,5}$

Interfacial thermal resistances $\left(R_{K}\right)$ associated with CNT-polymer and CNT-CNT interfaces present a fundamental limitation to the thermal conductivity enhancements. Both experiments and molecular dynamics simulations suggest $R_{K} \sim \mathrm{O}\left(10^{-8} \mathrm{~m}^{2} \mathrm{~K} \mathrm{~W}^{-1}\right)$ for CNT-polymer interfaces, ${ }^{4,6}$ and this resistance has been included phenomenologically in effective-medium theory (EMT) models for heat transport in CNT composites. ${ }^{7-9}$ In composites with higher CNT loading a description based on percolation might be required. ${ }^{10}$ However, modeling and simulations indicate that the CNT-CNT interface resistance also is $\mathrm{O}\left(10^{-8} \mathrm{~m}^{2} \mathrm{~K} \mathrm{~W}^{-1}\right)$ for small CNT-CNT separation. ${ }^{10,11}$ Other factors, such as CNT synthesis, quality, purification, dimensions, straightness, orientation, and dispersion, likely contribute to the modest nature of the enhancements and the high variability between studies; however, their effects are difficult to isolate from $R_{K}$.

Interfacial thermal resistances are known to increase significantly at low temperature; ${ }^{12}$ therefore, temperaturedependent $\kappa$-measurements allow investigation of increasing

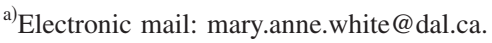

$R_{K}$ independent of changes in sample quality. Most experimental studies of CNT-polymer composites have focused around room temperature. One of the earliest thermal conductivity studies on a CNT/epoxy composite included a wide temperature range $(300$ to $30 \mathrm{~K})$ and reported that the $\kappa$-enhancement decreased as temperature decreased. ${ }^{13}$ More recently, $T$-dependent measurements from 300 to $2 \mathrm{~K}$ demonstrated changes in $\kappa$-enhancement such that at the lowest temperatures $\kappa$ of the composite was lower than that of the matrix. ${ }^{5}$ These observations are consistent with $\kappa$ limited by interfacial thermal resistance. In the present study we report temperature-dependent $\kappa$-measurements for single-walled CNT/polystyrene (SWCNT/PS) composites prepared by a method known to produce a more uniform distribution of SWCNTs on the micrometer scale within the polymer matrix. ${ }^{14}$ The electrical conductivity of the composites also is reported.

The SWCNTs used in these composites were produced by the high-pressure carbon monoxide method at Rice University (SWCNTs diameters around $1 \mathrm{~nm}$ and length $\sim 1 \mu \mathrm{m}){ }^{15}$ The raw SWCNTs were purified by thermal oxidation and $\mathrm{HCl}$ treatment, ${ }^{16}$ and contain less than 5 mass \% residual metal. The SWCNT/PS composites studied here contained either 0.75 or 1 mass \% SWCNTs in polystyrene $\left(M_{W}=126.4 \mathrm{~kg} \mathrm{~mol}^{-1}\right.$; polydispersity index $\left.=1.03\right)$ and were prepared by the coagulation method. ${ }^{14,17}$ In this solution-blending method a small amount of SWCNT $\left(\sim 0.1 \mathrm{mg} \mathrm{mL}^{-1}\right)$ was dispersed in $N, N$-dimethylformamide (DMF) with extensive sonication $(24 \mathrm{~h})$ followed by mixing with a PS/DMF solution and sonication for another $10 \mathrm{~s} .^{14,17}$ Whereas a limitation to dispersion quality in other solutionblended CNT composites is reagglomeration of the CNTs during solvent evaporation, ${ }^{18}$ coagulation overcomes this by dripping the SWCNT/polymer/DMF suspension into an excess of nonsolvent (water) to quickly produce the composite, before reagglomeration. ${ }^{17}$ After drying in vacuum $\left(130{ }^{\circ} \mathrm{C}\right.$, $24 \mathrm{~h}$ ), the raw composite material was consolidated by hot pressing under minimal pressure at $150{ }^{\circ} \mathrm{C}$.

The distribution of SWCNTs in composites prepared by the coagulation method has been characterized extensively, 


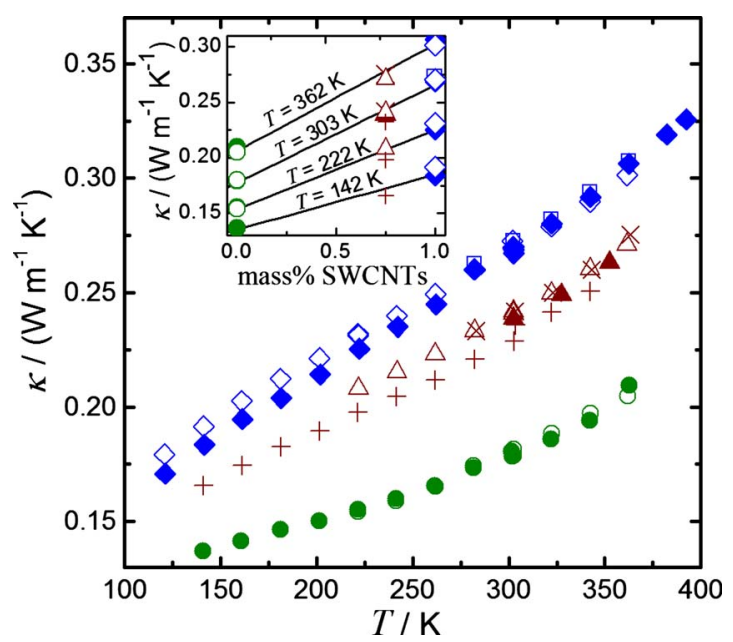

FIG. 1. (Color online) $\kappa$ vs $T$ for the PS matrix (2 samples: $\bullet, \bigcirc)$ and 0.75 mass \% SWCNT/PS (4 samples: $\triangle, \times,+, \mathbf{\Delta}$ ) and 1 mass \% SWCNT/PS (3 samples: $\diamond, \diamond, \square$ ). The inset shows $\kappa$ as a function of SWCNT content at selected temperatures.

including optical microscopy, scanning electron microscopy, UV-visible spectroscopy, small-angle x-ray scattering, and Raman imaging. ${ }^{17,19-23}$ Characterization shows that SWCNTs in such composites are present in small bundles, which are uniformly distributed on the micrometer scale. ${ }^{17,20,21}$ Before adding the polymer solution, a SWCNT sample was removed from the SWCNT/DMF suspension and atomic force microscopy was used to determine the mean diameter $(9.6 \mathrm{~nm})$ and the mean aspect ratio (35) of the SWCNT bundles.

Thermal conductivity was measured as a function of temperature using the longitudinal steady-state method using the thermal transport option of a Physical Property Measurement System (PPMS; Quantum Design, San Diego, CA). Measurements were conducted under vacuum $\left(10^{-4}\right.$ Torr $)$ and a radiation shield and corrective term were used to minimize radiative loss. Samples for thermal conductivity measurements were attached to disk-shaped, gold-plated copper leads using a thermally and electrically conductive adhesive (TraDuct 2902). The samples had uniform cross-sectional areas $\left(12-16 \mathrm{~mm}^{2}\right)$ and were approximately $1.5 \mathrm{~mm}$ thick.

Thermal conductivity results from approximately 140 to $360 \mathrm{~K}$ are shown in Fig. 1 (see Supplementary Information for data tables). ${ }^{24}$ Agreement between initial and subsequent measurements at room temperature indicates consistency in the sample and the thermal contacts within the assembly within this temperature range. Within the measured range the temperature dependence of $\kappa$ is approximately linear, with positive $d \kappa / d T$, and there is an increase in $d \kappa / d T$ with loading. In the range from 220 to $340 \mathrm{~K}$, the average slope increases from $(3.4 \pm 0.2) \times 10^{-4} \mathrm{~W} \mathrm{~m}^{-1} \mathrm{~K}^{-2}$ for the neat polystyrene to $(5.2 \pm 0.3) \times 10^{-4} \mathrm{~W} \mathrm{~m}^{-1} \mathrm{~K}^{-2}$ for the 1 mass \% SWCNT/PS composite.

Alternatively, thermal conductivity can be considered as a function of SWCNT loading. For the three loadings measured here $(0,0.75$, and 1 mass $\%)$, the thermal conductivity enhancement increased with SWCNT loading (Fig. 1, inset) and, at room temperature, reached $50 \%$ for the highest loading (1 mass \%). This enhancement is comparable to that reported by Yang et al. ${ }^{25}$ for MWCNT/PS composites, who note that the $\kappa$-enhancement is much larger with nanotubes

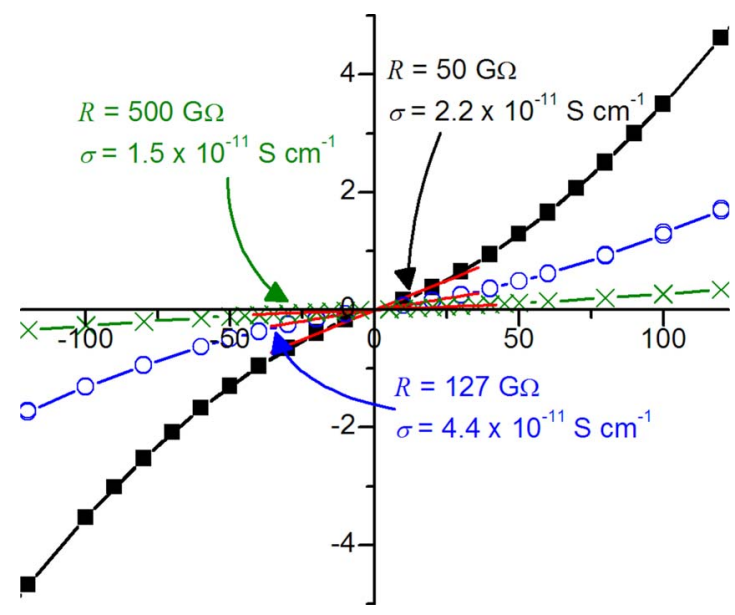

FIG. 2. (Color online) $I V$ curves for a 1 mass $\%$ SWCNT/PS sample measured along the pressing direction (ם) and the two perpendicular directions $(\bigcirc, \times)$. Sample resistance and conductivity, determined from the slope in the linear region and the sample dimensions, are indicated on the graph.

than with similar amounts of carbon nanofibers, graphite powder, or silver nanoparticles. ${ }^{25}$ The data suggest nonlinearity; however the effect is small for the low loadings studied here so linear fits are shown in accordance with EMT models. ${ }^{7-9}$ Other models, such as an EMT model including filler particle interactions, ${ }^{9}$ or approaches based on the Nielsen model, ${ }^{26,27}$ do provide for nonlinear behavior that might be more important at higher SWCNT loading.

The dependence of $\kappa$ on $T$ and SWCNT content is consistent with $R_{K}$ associated with the SWCNT/PS interfaces decreasing with increasing temperature (i.e., $R_{K}$ has a smaller negative effect on the thermal conductivity of the composite at higher temperature). The $d \kappa / d T$ values suggest this effect could lead to a cross-over at lower temperature, wherein the thermal conductivity of the composites would be lower than that of the neat PS, as we observed earlier for MWCNT/ epoxy composites. ${ }^{5}$ However, attempts to measure $\kappa$ for SWCNT/PS at lower temperatures $(<140 \mathrm{~K})$ were thwarted by thermal contact issues. Many adhesives were tested and in all cases a discontinuous, irreversible drop in $\kappa$ was observed when temperature was further reduced. This was attributed to a reduction in the quality of the thermal contact due to thermal expansion mismatch stress.

A recent study of SWCNT/PS composites by Peters $e t$ $a l^{28}$ suggested that a change in interfacial thermal resistance also should occur at the glass transition temperature $\left(T_{g}\right)$. The $T_{g}$ of the PS and composites studied here was $376 \mathrm{~K}$, as determined by differential scanning calorimetry (DSC; TA Instruments Q200). However, in contrast to the earlier $\kappa$ study using a modulated DSC technique ${ }^{28}$ we found that most samples exhibited an irreversible drop in $\kappa$ above $T_{g}$ (one exhibited no change).

Electrical conductivity also was determined through measurements of $I V$ curves using a Keithley 237 sourcemeasure unit (see supplementary information). ${ }^{24}$ For voltages up to $200 \mathrm{~V}$ (across a $3 \times 3 \times 1.5 \mathrm{~mm}^{3}$ sample) the current through PS samples was too low to measure. This indicated $\sigma_{\mathrm{PS}}<10^{-15} \mathrm{~S} \mathrm{~cm}^{-1}$, which is consistent with the expected, high resistivity of neat polystyrene. Larger, $V$-dependent currents were measured for the 0.75 and 1 mass \% SWCNT composites (e.g., Fig. 2 for a 1 mass $\%$ sample). For the 0.75 mass \% samples the results of upward 
and downward voltage sweeps were not consistent. However, those samples were clearly more conductive than the neat PS matrix and their conductivity was estimated from the currents at the largest voltages to be $10^{-14}$ to $10^{-13} \mathrm{~S} \mathrm{~cm}^{-1}$. The corresponding resistance is similar to the input impedance of the Keithley $237\left(10^{14} \Omega\right)$; therefore the measurement underestimates the sample resistance. For the 1 mass \% samples, $\sigma \sim 10^{-11}$ to $10^{-9} \mathrm{~S} \mathrm{~cm}^{-1}$ was determined from linear fits in the low- $V$, Ohmic region. The large change in $\sigma$ from 0 to 0.75 to 1 mass $\%$ suggests that the two loadings studied here fall within the relatively sharp jump in $\sigma$ that occurs around the percolation threshold.

Considering the 1 mass \% SWCNT/PS composites, the $\sigma$ values in the Ohmic region are similar in magnitude to those reported by Tchoul et al. ${ }^{29}$ for several SWCNT/PS composites but low in comparison to $10^{-6} \mathrm{~S} \mathrm{~cm}^{-1}$ reported for other 1 mass \% SWCNT/PS composites by the Winey group. ${ }^{23}$ Given the dramatic effect of dispersion, ${ }^{22}$ large variability between samples prepared separately is not unexpected. The nonlinear behavior of the $I V$ curves is welldescribed by the model of fluctuation-assisted tunneling, ${ }^{30}$ which has been applied to CNT networks ${ }^{30}$ and CNT/ polymer films. ${ }^{31}$ The bulk electrical measurements suggest some anisotropy in the samples. In one case the electrical conductivity was 100 times larger along the pressing direction and in other cases (e.g., for the sample shown in Fig. 2) the $\sigma$ 's along different directions are much closer. The differences could possibly be indicative of partial alignment, due to pressing, and variability the dispersion/SWCNT content on a millimeter scale for a sample near the percolation threshold. All $\kappa$ measurements were done along the pressing direction and there was no significant difference between the 1 mass \% samples despite the electrical conductivity differences. Therefore, possible anisotropy does not impact on interpretation of the $\kappa$ results.

In summary, thermal conductivity was measured as a function of temperature from 140 to $360 \mathrm{~K}$ for CNT loadings of $0,0.75$, and 1.0 mass $\%$ in SWCNT/PS composites, and was observed to increase with both temperature and SWCNT content. The $\kappa$-enhancement factor is reasonably constant above $300 \mathrm{~K}$ but decreases at lower temperatures. As interfacial thermal resistances increase at low temperature, this is consistent with the expectation that interfacial thermal resistance is a fundamental limitation on the $\kappa$ of $\mathrm{CNT} /$ polymer composites.

The authors thank M. McDowell and Professor I. Hill (Dalhousie) for useful discussions of electrical measurements, as well as access to equipment. This work was supported in part by the MRSEC program of the National Science Foundation under Award No. DMR05-20020, as well as NSERC and the Canada Foundation for Innovation, Atlantic Innovation Fund and other partners that fund the Facilities for Materials Characterization managed by the Institute for Research in Materials at Dalhousie University. M.B.J. acknowledges scholarship support from NSERC, the Killam Trusts, and the Sumner Foundation.

${ }^{1}$ M. Endo, M. S. Strano, and P. M. Ajayan, Top. Appl. Phys. 111, 13 (2008).

${ }^{2}$ K. I. Winey, T. Kashiwagi, and M. Mu, MRS Bull. 32, 348 (2007).

${ }^{3}$ W. Bauhofer and J. Z. Kovacs, Compos. Sci. Technol. 69, 1486 (2009).

${ }^{4}$ S. T. Huxtable, D. G. Cahill, S. Shenogin, L. Xue, R. Ozisik, P. Barone, M. Ursey, M. S. Strano, G. Siddons, M. Shim, and P. Keblinski, Nature Mater 2, 731 (2003)

${ }^{5}$ M. B. Jakubinek, M. A. White, P. C. P. Watts, and D. Carey, Mater. Res. Soc. Symp. Proc. 1022, II03-06 (2007).

${ }^{6}$ S. Shenogin, L. Xue, R. Ozisik, P. Keblinski, and D. G. Cahill, J. Appl. Phys. 95, 8136 (2004).

${ }^{7}$ C.-W. Nan, G. Liu, Y. Lin, and M. Li, Appl. Phys. Lett. 85, 3549 (2004).

${ }^{8}$ F. Deng, Q.-S. Zheng, L.-F. Wang, and C.-W. Nan, Appl. Phys. Lett. 90, 021914 (2007).

${ }^{9}$ F. Deng and Q.-S. Zheng, Acta Mech. Solida Sinica 22, 1 (2009).

${ }^{10}$ R. Haggenmueller, C. Guthy, J. R. Lukes, J. E. Fischer, and K. I. Winey, Macromolecules 40, 2417 (2007).

${ }^{11} \mathrm{H}$. Zhong and J. R. Lukes, Phys. Rev. B 74, 125403 (2006).

${ }^{12}$ E. T. Swartz and R. O. Pohl, Rev. Mod. Phys. 61, 605 (1989).

${ }^{13}$ M. J. Biercuk, M. C. Llaguno, M. Radosavljevic, J. K. Hyun, A. T. Johnson, and J. E. Fischer, Appl. Phys. Lett. 80, 2767 (2002).

${ }^{14}$ F. Du, J. E. Fischer, and K. I. Winey, J. Polym. Sci., Part B: Polym. Phys. 41, 3333 (2003).

${ }^{15}$ P. Nikolaev, M. J. Bronikowski, R. K. Bradley, F. Rohmund, D. T. Colbert, K. A. Smith, and R. E. Smalley, Chem. Phys. Lett. 313, 91 (1999).

${ }^{16}$ W. Zhou, Y. H. Ooi, R. Russo, P. Papanek, D. E. Luzzi, J. E. Fischer, M. J. Bronikowski, P. A. Willis, and R. E. Smalley, Chem. Phys. Lett. 350, 6 (2001).

${ }^{17} \mathrm{M}$. Mu, N. Clarke, R. J. Composto, and K. I. Winey, Macromolecules 42 , 7091 (2009).

${ }^{18}$ M. Moniruzzaman and K. I. Winey, Macromolecules 39, 5194 (2006).

${ }^{19}$ F. Du, R. C. Scogna, W. Zhou, S. Brand, J. E. Fischer, and K. I. Winey, Macromolecules 37, 9048 (2004).

${ }^{20}$ T. Kashiwagi, F. Du, K. I. Winey, K. M. Groth, J. R. Sheilds, S. P. Belanger, H. Kim, and J. F. Douglas, Polymer 46, 471 (2005).

${ }^{21}$ M. Mu and K. I. Winey, J. Phys. Chem. C 111, 17923 (2007).

${ }^{22}$ T. Kashiwagi, J. Fagan, J. F. Douglas, K. Yamamoto, A. N. Heckert, S. D. Leigh, J. Obrzut, F. Du, S. Lin-Gibson, M. Mu, K. I. Winey, and R. Haggenmueller, Polymer 48, 4855 (2007).

${ }^{23}$ M. Mu, A. M. Walker, J. M. Torkelson, and K. I. Winey, Polymer 49, 1332 (2008)

${ }^{24}$ See supplementary material at http://dx.doi.org/10.1063/1.3323095 for thermal conductivity data tables and additional detail on the $I V$ curves.

${ }^{25}$ Y. Yang, M. C. Gupta, J. N. Zalameda, and W. P. Winfree, Micro \& Nano Lett. 3, 35 (2008)

${ }^{26}$ E. H. Weber, M. L. Clingerman, and J. A. King, J. Appl. Polym. Sci. 88, 123 (2003).

${ }^{27}$ C. Guthy, F. Du, S. Brand, K. I. Winey, and J. E. Fischer, J. Heat Transfer 129, 1096 (2007)

${ }^{28}$ J. E. Peters, D. V. Papavassiliou, and B. P. Grady, Macromolecules 41, 7274 (2008)

${ }^{29}$ M. N. Tchoul, W. T. Ford, M. L. P. Ha, I. Chavez-Sumarriva, B. P. Grady, G. Lolli, D. E. Resasco, and S. Arepalli, Chem. Mater. 20, 3120 (2008).

${ }^{30}$ A. B. Kaiser and Y. W. Park, Synth. Met. 152, 181 (2005).

${ }^{31}$ V. Kažukauskas, V. Kalendra, C. W. Bumby, B. M. Ludbrook, and A. B Kaiser, Phys. Status Solidi C 5, 3172 (2008). 